\title{
O fazer dos estágios curriculares na formação em psicologia: uma experiência coletiva
}

\author{
The making of curricular internships in \\ psychology training: a collective experience
}

\section{Ana Aparecida Nascimento Martinelli Braga' ${ }^{1}$ Sylvia Maria Barreto da Silva² (1) Myla de Lima Arouca Oliveira 3 (1)}

\begin{abstract}
${ }^{1}$ Autora para correspondência. Escola Bahiana de Medicina e Saúde Pública (Salvador). Bahia, Brasil. anacidabraga@bahiana.edu.br
\end{abstract} 2,3Escola Bahiana de Medicina e Saúde Pública (Salvador). Bahia, Brasil. sylviabarreto@bahiana.edu.br, mylaarouca@bahiana.edu.br

RESUMO | O presente trabalho apresenta a concepção e o fazer dos estágios básicos e específicos do curso de Psicologia da Escola Bahiana de Medicina e Saúde Pública (EBMSP), instituição vocacionada para a saúde. Em seguida, descreve o funcionamento das referidas práticas, apresentando o contexto na qual elas estão inseridas e o processo de gestão do processo. Os estágios ocupam um lugar diferenciado na organização curricular do curso de Psicologia da EBMSP, pois oferecem aos discentes possibilidades de articulação entre teoria e prática de maneira crítica e reflexiva, conforme orientação das Diretrizes Curriculares Nacionais para os Cursos de Graduação de Psicologia. Os componentes fazem parte do eixo pré-profissionalizante e possibilitam a experiência em espaços e processos diversos de trabalho, nas duas ênfases do curso, Saúde e Trabalho. As atividades acontecem ao longo do quarto e quinto ano e são mediadas por vários participantes: corpo discente, corpo docente, coordenação e supervisor de campo. É a organização acadêmica e pedagógica dos estágios que permite a oferta de experiências práticas diferenciadas para o desenvolvimento de competências relacionadas ao fazer do Psicólogo. Observa-se que ao longo dos 20 anos do curso de Psicologia da EBMSP, os estágios estão em aprimoramento contínuo com foco na construção da identidade profissional do discente. Evidenciase, a partir da avaliação dos discentes, docentes e dos supervisores de campo que o egresso do curso de Psicologia da EBMSP, apresenta uma formação generalista, com competências práticas adquiridas para atuarem nos campos da saúde e trabalho com elevado nível técnico, integrados de forma crítica à sociedade, à realidade e ao mundo de trabalho.

PALAVRAS-CHAVE: Estágios. Psicologia. Formação.
ABSTRACT | This work presents the conception and implementation of basic and specific internships in the Psychology course at the Bahiana School of Medicine and Public Health (EBMSP), an institution dedicated to health. Then, it describes the functioning of these practices, presenting the context in which they are inserted and the process management process. The internships occupy a different place in the curricular organization of the Psychology course at EBMSP, as they offer students possibilities of articulation between theory and practice in a critical and reflective way, as guided by the National Curricular Guidelines for Psychology Undergraduate Courses. The components are part of the pre-professional axis and enable the experience in different work spaces and processes, in the two emphases of the course, Health and Work. The activities take place over the fourth and fifth year and are mediated by several participants: student body, faculty, coordination and field supervisor. It is the academic and pedagogical organization of the internships that allows the offering of different practical experiences for the development of skills related to the Psychologist's work. It is observed that throughout the 20 years of the Psychology course at EBMSP, the internships are in continuous improvement with a focus on building the student's professional identity. It is evident from the evaluation of students, teachers and field supervisors that the graduate of the Psychology course at EBMSP, presents a generalist training, with practical skills acquired to work in the fields of health and work with high technical level, integrated critically to society, reality and the world of work.

KEYWORDS: Internship. Psychology. Formation. 


\section{Introdução}

A formação de um curso de graduação em psicologia é muito ampla e complexa. Ao longo de cinco anos, sejam eles divididos em semestres ou blocos anuais, se faz necessário abranger desde reflexões epistemológicas e históricas da sua criação enquanto ciência, passando por eixos teóricos fundamentais, bases de pesquisa e reflexão social, até o desenvolvimento de práticas, contemplando as diversas psicologias.

Segundo o Relatório Final da Revisão das Diretrizes Curriculares Nacionais para os Cursos de Graduação de Psicologia (2018), apresentado no Art. $5^{\circ}$ da Minuta: "A formação em Psicologia deve ser presencial, generalista, multi e interdisciplinar, baseada na diversidade teórico-metodológica e na pluralidade dos seus campos de atuação, reconhecendo a identidade nacional, respeitando os contextos regionais e atendendo às diferentes necessidades dos indivíduos e populações, de forma inclusiva." Ainda nesse documento, os estágios curriculares são destacados como uma etapa fundamental da formação que tem orientação obrigatoriamente presencial e visa o desenvolvimento e a integração da teoria e prática ligados aos processos diferenciados de trabalho ofertados nas ênfases curriculares do curso e articulada às demandas sociais.

A ciência psicológica se caracteriza pela sua diversidade, trabalha com gente, e é feita também por gente (Bock, 2018). A inclusão faz parte dessa dinâmica, com vários objetos de estudos, várias abordagens teórico práticas, muitas técnicas diferentes e visões de mundo também. No mundo acadêmico, seja no ensino, na pesquisa e na extensão, quando se discute a Psicologia, a reflexão é sobre múltiplo, possibilidades, ainda que sempre atento ao singular, ao sujeito, ao indivíduo, inserido no seu contexto social.

Quando se pensa em estágio, identifica-se a prática, mundo do trabalho, atuação profissional. Onde trabaIha a Psicóloga, em que lugar está o Psicólogo? Mais uma vez as respostas são plurais, seja nos vários lugares já pensados: clínicas, empresas, ambulatórios, hospitais, comunidades, seja em quaisquer outros que ainda serão inventados e criados. Mas é importante destacar que em todos eles há dois eixos norteadores fundamentais, ética e promoção de saúde.
Portanto, a Psicologia é uma profissão de saúde, e, então, a proposta do presente artigo é descrever as práticas de estágio, sejam na ênfase trabalho ou saúde, na Escola Bahiana de Medicina e Saúde Pública (EBMSP) que funcionam sempre em torno deste propósito, contemplando a diversidade.

\section{Organização curricular do curso de Psicologia}

Em 2020, o curso de Psicologia da Escola Bahiana de Medicina (EBMSP) completou 20 anos. Sua autorização ocorreu em 1998, mas foi implantado em 2000 com o ingresso da primeira turma. A proposta pedagógica estava de acordo com as determinações do Currículo Mínimo para o ensino superior, parâmetros vigentes à época. No ano da implementação, já se discutia uma nova proposta de formação em Psicologia, com base nas Diretrizes Curriculares Nacionais (DCN's) para graduação em psicologia . Com isso, o projeto inicial foi modificado a partir de uma ampla discussão e os estágios básicos e específicos foram organizados e inseridos com base nas DCN's.

O propósito do curso de Psicologia da EBMSP é a formação de profissionais aptos para atuar em diferentes contextos considerando as necessidades sociais, os direitos humanos e cônscios de que sua intervenção deve estar voltada para a promoção da saúde e da qualidade de vida de indivíduos, grupos, organizações e comunidades. Para isso, comunidade acadêmica conta com uma organização curricular generalista e abrangente, que possibilita atuarem nos campos da saúde e trabalho com elevado nível técnico, integrados de forma crítica à sociedade, à realidade e ao mundo do trabalho.

Para que se possa compreender melhor o contexto no qual os estágios estão inseridos, é importante explicar um pouco mais sobre o funcionamento do curso. Podemos dividi-lo em três grandes blocos, o bloco inicial abrange do primeiro ao sexto semestre, o segundo bloco acontece no sétimo e oitavo semestres e o bloco final diz respeito ao último ano do curso.

No bloco inicial, o discente já começará a desenvolver o raciocínio clínico e crítico, a partir de discussão de casos em pequenos grupos com professores tutores, no eixo temático Ser Humano e Ciclo de Vida, 
desde o início da vida, passando pela infância, infância escolar, adolescência, mundo dos adultos e envelhecimento. Durante esses seis semestres, também cursará componentes curriculares transversais que fazem parte do eixo temático Intervenções em Psicologia e proporcionam ao discente o estudo de diferentes técnicas e instrumentos de atuação do psicólogo que envolve modelos de observação, entrevista e manejo de grupo. A partir das experiências nos referidos eixos temáticos ocorre o desenvolvimento de competências vinculadas ao protagonismo e saber autoral discente.

Ao chegar no sétimo semestre do curso há uma virada importante, pois o discente se aproxima ainda mais da realidade de atuação do psicólogo, e se depara ao vivo, com os "casos" discutidos nos semestres anteriores. Os professores acompanham seus discentes, em grupos menores, nos reais campos de estágio básico, em quatro frentes de atuação, que circulam em rodízio ao longo ao longo de dois semestres ( $7^{\circ}$ e $8^{\circ}$ semestres), sejam na ênfase em saúde (atenção primária, secundária e terciária), seja na ênfase de trabalho e organização.

No quinto ano, o discente escolhe uma ênfase e desenvolve uma prática de estágio específico, já com autonomia adquirida, orientado pelo psicólogo de campo de uma instituição parceira e supervisionado pelo professor da EBMSP.

\section{Funcionamento dos Estágios}

As atividades de estágio curricular em Psicologia realizadas no $7^{\circ}, 8^{\circ}, 9^{\circ}$ e $10^{\circ}$ semestres ocorrem em instituições parceiras e conveniadas com a EBMSP, são regidas pela Lei Federal $n^{\circ} 11.788$, de 25 de setembro de 2008, ratificada pela Portaria Federal de $n^{\circ} 305$, publicada no Diário Oficial de 26 de fevereiro de 2014 e desenvolvidas em diferentes contextos de práticas, com a finalidade de enriquecer a formação discente.

Os estágios são devidamente planejados, executados, acompanhados e avaliados, em consonância com o Projeto Pedagógico do Curso (PPC) e o corpo discente experimenta a práxis no mundo laboral, participando e intervindo de forma a integrar as competências conceituais, procedimentais e atitudinais desenvolvidas ao longo do curso.
A proposta das atividades de estágio básico na instituição de ensino superior que trata esse relato, configura-se como práticas interdisciplinares em contextos diferenciados que proporcionam ao discente a articulação entre a teoria e formas de atuação profissional, além do desenvolvimento de competências gradual inerentes ao fazer do psicólogo.

No eixo temático das práticas pré-profissionalizantes é oportunizado ao discente do $7^{\circ}$ e $8^{\circ}$ semestres a modalidade de ensino do Internato de Psicologia que compreende a vivência em quatro cenários diferentes que exigem intervenções psicológicas de natureza relativa a diagnóstico, planejamento e execução. Os componentes curriculares (Psicologia na Atenção Primária, Secundária e Terciária à Saúde e Psicologia no Trabalho e na Organização) estão organizados numa proposta didática inovadora baseada no aprendizado em serviço, que pressupõe a observação da prática, a discussão de casos reais e o desenvolvimento de intervenções, sob a supervisão de docentes em tempo real no campo que acompanham o máximo de 12 discentes, por turma, garantindo a responsabilidade técnica das atividades realizadas. Esse processo de aprendizagem ocorre em quatro rodízios alternados de 10 semanas, cada um, com etapas que compreende um período de preparação, desenvolvimento de intervenções nos quatro cenários de prática oferecidos por cada componente curricular e avaliação da atuação, em uma carga horaria semanal de 12 horas, totalizando 120 horas por rodízio e 480 horas ao final de todo o processo.

O diferencial desta modalidade pedagógica apresenta ao discente a possibilidade de ampliação dos níveis de interação com atores sociais dos contextos que está inserido e complexidade do processo formativo, convocando para uma atuação mais autônoma com a mediação do docente na construção da aprendizagem. Os objetivos gerais do Internato, baseado nas DCNs publicada em março de 2011, são: oferecer oportunidades para ampliar, integrar e aplicar os conhecimentos adquiridos nos anos anteriores do curso de graduação; permitir melhor treinamento em técnicas e habilidades indispensáveis ao exercício do fazer do psicólogo; promover o aperfeiçoamento ou a aquisição de atitudes adequadas à prática profissional; desenvolver a capacidade de identificar, resolver ou bem encaminhar os problemas de saúde da população a que vai servir; possibilitar a prática da assistência integrada em contextos que envolvem 
equipes multiprofissionais; estimular o interesse pela promoção e preservação da saúde e da qualidade de vida de sujeitos e organizações; desenvolver a consciência das responsabilidades, limitações e deveres éticos do psicólogo, perante o paciente, a instituição e a comunidade; desenvolver a ideia da necessidade de aperfeiçoamento profissional continuado. Embora guiado pelos objetivos gerais, a experiência de aprendizagem se singulariza a cada rodízio ofertado pelos distintos componentes curriculares que compõe a modalidade do Internato, nas ênfases do curso.

Para garantir uma gestão integrada e participativa, a Comissão Pedagógica do Internato (COPI), composta pela coordenação do curso, coordenação do internato, docentes dos componentes curriculares e supervisão pedagógica, foi criada e trata das questões relativas ao processo de ensino e aprendizagem através de reuniões periódicas e/ou extraordinárias. Cabe ao coordenador do internato organizar, acompanhar e atualizar o programa, além de intermediar a comunicação com os campos parceiros e deliberar sobre as solicitações do discentes e docentes.

O processo avaliativo no Internato é realizado em níveis de complexidade distintos, crescentes e processual, a partir de intervenções individuais e em grupo, respeitando as peculiaridades de cada serviço. É oportunizado ao docente e ao discente identificar o nível do desenvolvimento das competências gerais e especificas previstas. São produtos concretos do aprendizado do discente, os projetos de intervenção elaborados a partir de diagnóstico e execução de práticas, elaboração e análise de casos clínicos que fazem sentido ao seu percurso formativo. Os feedbacks são valorizados como forma de avaliação utilizada com maior frequência, visto que a convocação do discente para demonstrar o saber fazer é contínuo.

Os componentes referentes ao Internato são pré-requisitos para ingressar no estágio específico e possibilita um processo de transição entre o papel de estudante e a posição de psicólogo em formação, favorecendo o desenvolvimento do protagonismo, de uma postura ética e comprometida com a necessidade de cada contexto social, com os direitos humanos e com a diversidade.
As atividades de Estágio Específico em Psicologia, na EBMSP são realizadas no $9^{\circ}$ e $10^{\circ}$ semestres, têm como finalidade enriquecer a formação do discente, na modalidade de estágio obrigatório e são desenvolvidas em diferentes contextos, de acordo com uma das ênfases escolhidas pelo discente.

A referida experiência é acompanhada por supervisão de campo e supervisão acadêmica. Esta última é dimensionada com uma carga horária de oito horas semanais, permitindo ao supervisor encontrar-se semanalmente com seu grupo de estagiários para quatro horas de atividades teórico-práticas e contempla, também, em outro turno de quatro horas, a visita do supervisor acadêmico ao local de estágio do estudante e supervisões individuais, quando necessário. Cada supervisor tem, no máximo, 10 estagiários por semestre letivo, permitindo a troca não somente com o professor, mas também entre os pares. Os encontros de supervisão acadêmica são registrados na caderneta eletrônica e as atividades diárias do estudante são registradas na caderneta de campo. Esse acompanhamento permite uma administração transparente da gestão do conhecimento no estágio, estando aberta entre o docente e o discente.

O Estágio Específico é a atividade curricular orienta$\mathrm{da}$, na qual o discente exercita habilidades na construção de competências para a prática profissional. Esse é o momento no qual se constitui a atividade de exercício real do papel de psicólogo. O processo é acompanhado não só pelos supervisores, mas também pela Coordenação de Estágio e Coordenação de Curso. Cabe ao coordenador de estágio articular e mediar as relações entre a EBMSP e os campos de estágio específico por meio de visitas e reuniões com vistas a assegurar o desenvolvimento das competências estabelecidas no planejamento das atividades de estágio. Esse trabalho envolve corpo discente e o processo da escolha do estágio, corpo docente, o acompanhamento das atividades de supervisão e visitas aos campos de prática, bem como instituições parceiras para a promoção de abertura de vagas e garantia de um aprendizado para o discente.

O processo de escolha pela ênfase do estágio tem início no final do $8^{\circ}$ semestre, a partir da entrega, ao estudante, da ficha de escolha de estágio, contendo 
as duas ênfases do curso e, entre estas, as áreas de atuação, para que ele defina as suas prioridades, por ser o discente em potencial do componente curricular Estágio Específico no semestre seguinte. A partir dessas informações, a Coordenação de Estágio realiza um levantamento da situação de escolhas, para que sejam organizados os grupos de supervisão e auxiliada a articulação de vagas com os campos. Ainda nesse semestre, e durante o período de recesso escolar, os discentes participam de processos seletivos e, em seguida, aguarda-se o período de matrícula.

O estudante que desejar participar de processos seletivos em campos novos, ainda não conveniados com a EBMSP, deve solicitar, junto à Secretaria Acadêmica, a avaliação da unidade concedente para fins de realização de estágio, apresentando o formulário próprio para esse fim, devidamente preenchido e assinado. Fica estabelecido o prazo de até dez dias úteis para a avaliação da referida unidade, a partir da qual, se de acordo, é celebrado o termo de convênio entre a EBMSP e a nova instituição parceira.

Após realização da matrícula no componente curricular de Estágio Específico, automaticamente, o discente é coberto por uma Apólice de Seguros. Em seguida, a Secretaria providencia o Termo de Compromisso de Estágio (TCE) para cada discente, em três vias, estabelecendo a relação de estágio entre a EBMSP, o discente e a instituição parceira. O discente deve iniciar o semestre letivo devidamente matriculado e inserido no campo de estágio. Caso, por alguma circunstância, isso não seja possível, só será permitida a entrada do discente até o final da $2^{a}$ semana de supervisão e atividades práticas.

O estudante do Estágio Específico deverá cumprir dois semestres de, no mínimo, 360 horas cada um, que constituem os componentes curriculares Estágio Específico I e Estágio Específico II. Essa carga horária total é dividida em 288 horas de prática no Campo (16h semanais) e 72 horas de supervisão acadêmica (4h semanais), tendo em vista 18 semanas de atividades acadêmicas semestrais.

O processo de avaliação do estágio específico envolve aspectos qualitativos e quantitativos. A Secretaria elabora uma caderneta de campo, a ser entregue pelo professor-supervisor ao psicólogo que acompanhará o discente no campo, que contém: um Formulário de Programa das Atividades de Estágio, planilhas de acompanhamento da frequência do estagiário e um Formulário de Avaliação do Desenvolvimento do Estagiário, a ser preenchido pelo psicólogo do campo. Ao professor da Bahiana cabe definir outros formatos de avaliação, incluindo por exemplo Relatório de Estágio e Instrumento de Avaliação Atitudinal.

A organização acadêmica e pedagógica dos estágios descritas anteriormente proporciona a articulação entre teoria e prática; desenvolvimento de profissionais éticos, com domínio teórico e prático-profissional; inserção do discente em uma reflexão crítica e coletiva; participação e reflexão sobre situações acadêmicas para compreensão e atuação em situações contextualizadas e o desenvolvimento de competências conceituais, procedimentais e atitudinais essenciais ao exercício profissional do psicólogo.

Anualmente, é realizada uma atividade de Extensão intitulada Seminário de Preparação para Estágio e Trabalho (SEPET), cujo objetivo é preparar os estudantes para a escolha de estágio em uma das ênfases (Saúde e Trabalho) oferecidas pelo curso de Psicologia, bem como para o encontro com o mundo do trabalho, através da apresentação dos campos de estágio, práticas desenvolvidas e supervisores envolvidos, identificando suas possibilidades e desejos e favorecendo a reflexão sobre os projetos de futuro. Por meio da exposição e do compartilhamento de práticas de intervenção, pretende-se, com o SEPET, aprimorar competências necessárias ao desempenho de uma prática profissional ética e humanizada, em diferentes contextos da sociedade. Em 2019, a $11^{a}$ edição do evento foi organizada por estagiárias do último semestre, juntamente com a coordenação de estágio. Houve a participação do NAPP (Núcleo de Atendimento Psicopedagógico) refletindo sobre a importância do cuidar de si para cuidar do outro, presença de psicólogas de campos de estágio, compartilhando o desenvolvimento das práticas, juntamente com as estagiárias; Conselho Regional de Psicologia, ressaltando a importância do trabalho ético, bem como rodas de conversas, manejadas pelos próprios estagiários, apresentando as atividades desenvolvidas nos diversos cenários de prática. 


\section{Considerações Finais}

Em síntese, a gestão e operacionalização dos estágios no curso de Psicologia da EBMSP estão em constante aprimoramento. Fica evidente a cada novo ciclo de abertura de práticas, a relevância do trabalho integrado em equipe e a necessidade de mantermos a articulação entre os atores envolvidos no referido processo: discentes, docentes e parceiros de campo. Estes últimos, sinalizam o amadurecimento dos nossos discentes, a partir de uma postura ética e comprometida, com conteúdos consolidados e habilidades procedimentais seguras. Observamos que o modelo instituído das práticas pré-profissionalizantes oferecidos pelos estágios básicos e específicos favorece o processo de construção da identidade profissional e desenvolvimento pessoal do discente. Assim, é notório como o trabalho construído a partir da organização curricular por competências e que favorece o contato do discente com atividades práticas desde o início do curso, tem apresentado os resultados esperados e está em consonância com o perfil do egresso, definido no PPC, ou seja, estudantes aptos para atuarem com autonomia e protagonismo em diferentes contextos, promovendo saúde e qualidade de vida de indivíduos, grupos, organizações e comunidades de forma crítica e atento à dimensão social e à preservação dos direitos humanos. Além de agirem como agente transformador na construção de uma sociedade mais democrática e menos desigual, trabaIhando nas dimensões psicossocial, psicodinâmica, institucional e comunitária.

\section{Contribuições das autoras}

Braga, AANM; Silva, SMB; Oliveira, MLA participaram de todas as etapas: concepção e delineamento; revisão de literatura; apresentação e discussão dos resultados; considerações finais.

\section{Conflitos de interesses}

Nenhum conflito financeiro, legal ou político envolvendo terceiros (governo, empresas e fundações privadas) foi declarado para nenhum aspecto do trabalho submetido (incluindo mas não limitando-se a subvenções e financiamentos, conselho consultivo, desenho de estudo, preparação de manuscrito, análise estatística, etc).

\section{Referências}

Bock, A. M. B., Teixeira, M. L. T., \& Furtado, O. (2018). Psicologias: uma introdução ao estudo de psicologia (15a ed.). Saraiva.

Conselho Federal de Psicologia (2018). Ano da formação em psicologia: revisão das diretrizes curriculares nacionais para os cursos de graduação em psicologia. Conselho Federal de Psicologia/Associação Brasileira de Ensino de Psicologia/Federação Nacional dos Psicólogos. https://site.cfp.org.br/wp-content/uploads/2018/07/ RELAT\%C3\%93RIO-FINAL-REVIS\%C3\%830-DASDIRETRIZES-CURRICULARES-NACIONAIS-PARA-OS-CURSOSDE-GRADUA\%C3\%87\%C3\%830-EM-PSICOLOGIA.pdf

Escola Bahiana de Medicina e Saúde Pública (2015). Projeto Político Pedagógico do Curso de Psicologia da Escola Bahiana de Medicina e Saúde Pública. BAHIANA. http://www.bahiana. edu.br/CMS/Uploads/Projeto-Politico-PedagogicoPsicologia-Bahiana-2015.pdf

Escola Bahiana de Medicina e Saúde Pública (2020a). Manual de Estágio em Psicologia da Escola Bahiana de Medicina e Saúde Pública. AVA/BAHIANA. https://ava.bahiana.edu.br/ moodle $/ \mathrm{mod} /$ resource/view.php?id=82548

Escola Bahiana de Medicina e Saúde Pública (2020b). Manual do Internato de Psicologia da Escola Bahiana de Medicina e Saúde Pública. AVA/BAHIANA. https://ava.bahiana.edu.br/ moodle/pluginfile.php/465958/mod_resource/content/1/ MANUAL\%20DO\%2OINTERNATO\%202020.pdf 